\title{
Inhibition of return for the length of a line?
}

\author{
LORI FRANCIS and BRUCE MILLIKEN \\ McMaster University, Hamilton, Ontario, Canada
}

\begin{abstract}
Inhibition of return is most often measured using an exogenous spatial cuing method. The experiments presented here follow up on a small number of studies that have examined whether a similar effect occurs for nonspatial stimulus attributes. In Experiments 1 and 2, the task was to identify a target line as either short or long. In this context, targets on valid trials were of the same length as that of a preceding cue, whereas targets on invalid trials were of a different length than that of a preceding cue. The results were similar to those in spatial orienting studies in that responses were slower for valid than for invalid targets only at stimulus onset asynchronies (SOAs) longer than 300 msec. In Experiment 3, the stimuli were the same but the task was to detect the onset of the target line. This task change resulted in slower responses for valid than for invalid targets at all SOAs. A similar result was observed in Experiment 4, in which validity was defined by color rather than line length, and the task was to identify the target color. The discussion centers on an opponent process approach to interpreting cuing effects, and consequent difficulties in distinguishing spatial and nonspatial cuing effects based on their time course.
\end{abstract}

The orienting of attention is pervasive in human behavior and the subject of a great deal of contemporary research in experimental psychology. The spatial cuing method is among the most commonly used to study orienting processes (Posner, 1980). In this method, an attentional cue and then a target are presented to participants in succession. The cue is presumed to elicit a shift of attention to one particular location in space, either because it provides predictive information about the most likely location for a subsequent target or because it captures attention automatically (Jonides, 1981). Cuing effects are defined by differences in performance for targets that appear at the cued location and targets that appear at an uncued location.

Although much has been learned from use of the spatial cuing method, an issue that remains understudied is whether empirical effects revealed in spatial cuing studies reflect orienting processes that are specific to the dimension of space. In this article, we address this issue with reference to an effect known as inhibition of return (Posner \& Cohen, 1984). Four experiments are described in which cues and targets were either line segments or colored rectangles presented centrally. One critical result was that responses were often slower for validly cued than for invalidly cued targets. Given that the tasks did not involve spatial orienting processes in any obvious way, this result suggests that the processes responsible for inhibition of return may not be specific to the domain of space. A second critical result is that the time course of these cuing effects depended both on the stimulus at-

This research was supported by a Natural Science and Engineering Research Council (NSERC) grant awarded to B.M., and by an NSERC postgraduate scholarship awarded to L.F. L.F. is now in the Department of Psychology at St. Mary's University, Halilfax. Correspondence should be addressed to B. Milliken, Department of Psychology, McMaster University, Hamilton, ON L8S 4K1, Canada (e-mail: millike@ mcmaster.ca). tribute that defined cue validity and on the nature of the task. To accommodate these results, a theoretical framework with components that are sensitive to task and stimulus variables is offered. To set the experiments in context, a brief review of the inhibition of return literature is provided below (see also Taylor \& Klein, 1998b).

\section{Inhibition of Return and Spatial Cuing}

Posner and Cohen (1984) reported a series of experiments in which a central box was flanked to the left and right by two peripheral boxes. The first critical event on a trial was an attentional cue, which consisted of the brightening of one of the two peripheral boxes. At varying stimulus onset asynchronies (SOAs) following the cue, a target appeared inside one of the three boxes. Participants were to press a response key as quickly as possible after detecting the onset of the target. When the target followed the cue by less than approximately $300 \mathrm{msec}$, responses were faster for targets at the cued location than for targets at the uncued location. However, at longer cue-target SOAs, responses were slower for targets at the cued location than for targets at the uncued location, the effect now commonly called inhibition of return.

Posner and Cohen suggested that attention is initially attracted to the periphery by the cue, which explains the facilitation effect observed on short SOA trials. However, if the target does not appear shortly after the cue, then attention is disengaged from the cued location and shifted back to the central box before onset of the target. One explanation for the inhibition of return effect observed on these longer SOA trials focuses on this disengage process. In particular, the process of disengaging attention could leave behind an inhibitory tag that makes it difficult to return attention to the same location. Indeed, some authors have suggested that the processes responsible for the inhibition of return effect facilitate visual search by 
favoring novel locations over those that attention has already visited (Klein, 1988; Posner \& Cohen, 1984). For future reference, we label this hypothesis the disengage account of inhibition of return. An alternative hypothesis is that the process that causes inhibition of return is independent of the disengage process. Instead, disengagement of attention may appear to be related to inhibition of return because, prior to disengagement, inhibition of return is masked by an opposing facilitatory effect of the cue (Danziger \& Kingstone, 1999; Klein, 2000; Mondor, 1999; Tipper et al., 1997). We label this hypothesis the opponent process account of inhibition of return. The distinction between these two hypotheses takes on particular importance in later discussions of nonspatial cuing effects.

Although initial studies indicated that inhibition of return occurs only with exogenous attentional cues (Posner \& Cohen, 1984), it was soon discovered that inhibition of return can occur without a change in luminance at the cued location. Posner, Rafal, Choate, and Vaughan (1985) used a central arrow cue to direct attention to the cued location. Observers were instructed to move their eyes as well as their attention to the cued location. An increment in luminance then occurred at the central box, presumably pulling attention away from the previously cued location and back to center. A target then followed with equal probability in either of the peripheral boxes. Observers were to detect the onset of the target by a keypress. Responses to targets at the cued location were slower than to targets at the uncued location. Thus, it appeared that inhibition of return could occur in an endogenous cuing task as long as attention shifts were accompanied by eye movements. However, Rafal, Calabresi, Brennan, and Sciolto (1989) showed that inhibition of return can also be observed following an instruction to prepare an eye movement to a peripheral location in response to a central cue, but then to "cancel" that prepared eye movement. This result suggests that neither an abrupt luminance change nor an eye movement to the cued location is necessary to produce inhibition of return. Instead, Rafal et al. suggested that both of these conditions lead to inhibition of return because they elicit oculomotor activation.

\section{Inhibition of Return and Dimensions Other Than Space}

Although the series of studies just described led researchers to attribute inhibition of return to activation of the oculomotor system, it remains possible that inhibition of return instead reflects a more general property of the interaction between perceptual and motor systems. If this is the case, then one might expect similar effects to occur for stimulus attributes other than spatial location.

Law, Pratt, and Abrams (1995) investigated inhibition of return in a color detection task. Participants were presented with a color patch cue, followed by a neutral color patch, and then a target patch that either matched or mismatched the color of the cue. Participants were indeed slower to detect the onset of a target color patch when the same color had been presented as a cue (valid trials) than when a different color had been presented as a cue (invalid trials; see also Fox \& DeFockert, 2001; Lupiáñez \& Tudela, 1999; but see Kwak \& Egeth, 1992). Although this effect was somewhat smaller in magnitude (5-6 msec) than the effects often observed in spatial orienting studies, Law et al. described it as an inhibition of return effect for a stimulus attribute other than spatial location.

A conclusion that might be drawn from the Law et al. (1995) study is that there is nothing special about space insofar as measuring inhibition of return is concerned. Rather, inhibition of return may occur for any number of different perceptual attributes. However, there are counterarguments to this broad claim that merit consideration. For example, exogenous spatial cuing effects are often SOA dependent, with null or facilitatory cuing effects occurring at short SOAs, and inhibition of return emerging only at longer SOAs. Yet, Law et al. measured performance in their color detection task using only a relatively long cue-target SOA $(1,800 \mathrm{msec})$. Taylor and Klein (1998a) have since measured performance in a color detection task, similar to that of Law et al., and found that responses were slower for valid targets at all SOAs, rather than just at longer SOAs. Fox and DeFockert (2001) also measured color cuing effects in a detection task, and found that responses were slower for valid than for invalid targets at short cue-target SOAs, with this effect dissipating with increasing cue-target SOA. The point to note is that neither of these studies produced a time course for cuing effects that matched that observed in many spatial cuing studies. To the extent that the time course of cuing effects is used as a signature property of underlying attentional processes, these findings (Taylor \& Klein, 1998a; Fox \& DeFockert, 2001) suggest that validity effects in exogenous spatial orienting studies may have a different cause than validity effects in exogenous nonspatial cuing studies (see also Kraut \& Smothergill, 1978).

Several recent auditory orienting studies have also challenged the view that inhibition of return is a strictly spatial phenomenon. Mondor, Breau, and Milliken (1998) presented participants with cue and target tones separated by a variable SOA. For some participants, the task was to detect the onset of the target tone, while for other participants, the task was to indicate the location or frequency of the target tone. The cue and target tones were either the same or different in spatial location, or the same or different in frequency. In all cases, there was a response time (RT) advantage at short SOAs for valid trials. However, at long SOAs there was a RT disadvantage for valid trials. Note that this pattern of validity effects matches that often found in spatial orienting studies. That this pattern occurred both when validity was defined by location and when validity was defined by frequency suggests that inhibition of return in audition is not restricted to the dimension of space. Furthermore, Mondor and Lacy (2001) have since reported a similar pattern of results with validity defined by auditory duration, inten- 
sity, or timbre. Together, these results demonstrate that inhibition of return effects for auditory stimuli generalize to stimulus properties other than those thought to be dominant in guiding auditory attention (i.e., space or frequency; see Mondor, Zatorre, \& Terrio, 1998). Yet, as mentioned, the evidence that inhibition of return for visual stimuli generalizes to stimulus properties other than those thought to be dominant in guiding visual attention (i.e., space) is much less clear.

\section{The Present Study}

The purpose of the present study was to examine further whether inhibition of return occurs in nonspatial visual cuing tasks. In Experiments 1 and 2, a straight line centered in the stimulus display served as a cue stimulus, and a second straight line served as a target stimulus. Both the cue and target lines were either short or long, and participants were required to identify the length of the target line only. In Experiment 3, again straight lines centered in the stimulus display served as cues and targets, but participants were required simply to detect the onset of the target line. In Experiment 4, colored rectangles served as cues and targets, and participants were required to identify the target color. In all of the experiments, the relation between cue and target (valid/invalid) and the cue-target SOA were the key independent variables. One issue of interest was whether this type of task would provide any evidence for slowed responding on valid trials relative to invalid trials. A second issue of interest was whether the time course of cuing effects would follow that commonly observed in exogenous spatial orienting studies - that is, whether responses for valid targets would be slower than those for invalid targets only at relatively long cue-target SOAs.

\section{EXPERIMENT 1}

Participants in this experiment were presented with a horizontal line in two consecutive displays with varying temporal intervals between the displays. Both the first line (the cue) and the second line (the target) could be either short or long. The task was to identify the length of just the target line. If cuing effects in this task have the same cause as cuing effects in an exogenous spatial orienting task, then one might expect them to be sensitive to the cue-target SOA, with slower responses to valid targets (targets of the same length as the cue) than to invalid targets emerging only at the longer SOAs.

Two between-subjects variables were included in the experiment to examine the stability of the time course of cuing effects in this task. One variable involved task difficulty. Prior work using a spatial cuing procedure has revealed that task difficulty can alter the time course of cuing effects (Lupiáñez, Milliken, Solano, Weaver, \& Tipper, 2001). We included a task difficulty manipulation here simply to examine whether a similar result would occur in this nonspatial task. The second between- subjects variable concerned the proportion of trials in each of the SOA conditions. Two recent studies have shown that temporal expectancy can modulate the time course of spatial cuing effects under some conditions (Milliken, Lupiáñez, Roberts, \& Stevanovski, in press; Mondor, 1999). Again, we were interested in whether temporal expectancy might modulate cuing effects in this nonspatial task.

\section{Method}

Participants. Eighty undergraduate students with a mean age of 21 years participated for course credit. Participants were recruited from an introductory psychology course at McMaster University. Twenty participants were assigned to each of four groups. ${ }^{1}$ All participants reported normal or corrected-to-normal visual acuity.

Apparatus and Stimuli. Stimuli were presented on a SVGA color monitor that was connected to an IBM-compatible Pentium computer. The experiment was carried out using Micro Experimental Laboratory (MEL) software (Schneider, 1988). Participants sat facing the monitor at a viewing distance of approximately $57 \mathrm{~cm}$.

The background of the screen remained black for the duration of the experiment. The fixation point was a plus sign $\left(0.6^{\circ}\right.$ of visual angle horizontally $\times 0.7^{\circ}$ of visual angle vertically) in the center of the screen. The stimuli used in this experiment were two white lines. One line was defined as long and the other was defined as short. The short and long lines subtended $0.2^{\circ}$ vertically. The horizontal length of the short and long lines varied across groups, as described in the Procedure section below.

Procedure and Design. The experiment consisted of 1 block of 16 practice trials followed by 20 blocks of 16 experimental trials. At the beginning of each block, the message "Press $b$ to begin block" appeared on the screen. Once the block was initiated, the following sequence of events occurred for each trial: (1) a fixation cross appeared until participants pressed the space bar; (2) a 250-msec blank field; (3) a 100-msec presentation of the cue; (4) a variable blank interval of $200,500,800$, or $1,100 \mathrm{msec}$ (resulting in SOAs of $300,600,900$, and $1,200 \mathrm{msec}$ ); (5) presentation of the target for $100 \mathrm{msec}$; and (6) a keypress response by participants. This sequence is displayed in Figure 1. Participants were instructed to keep their eyes and attention focused on the fixation cross prior to the trial. The task was to indicate whether the target line was short or long. Responses were recorded by means of a keypress. Half of the participants responded "short" with their dominant hand, while half responded "long" with their dominant hand. The keypress triggered the onset of the fixation cross for the next trial. RTs were measured as the latency between the onset of the target display and the keypress, and participants were asked to respond as quickly and accurately as possible.

Two within-subjects variables were manipulated. One of these variables was validity, which consisted of two levels. In the valid condition, the cue and the target lines were the same length (both short or both long). In the invalid condition, the cue and target lines were of different lengths (short cue, long target or long cue, short target). Half of the trials in the experiment were valid and half were invalid. The other within-subjects variable was SOA. This variable had four levels: $300,600,900$, and 1,200 msec.

Two between-subjects variables were also manipulated. The difference in length between the short and long lines was smaller in the difficult discrimination condition than in the easy discrimination condition. For the easy discrimination groups, the short line subtended $1.75^{\circ}$ horizontally and the long line subtended $6.75^{\circ}$ horizontally. For the difficult discrimination groups, the short line subtended $3.00^{\circ}$ horizontally, while the long line subtended $4.25^{\circ}$ horizontally. The second between-subjects variable concerned the 


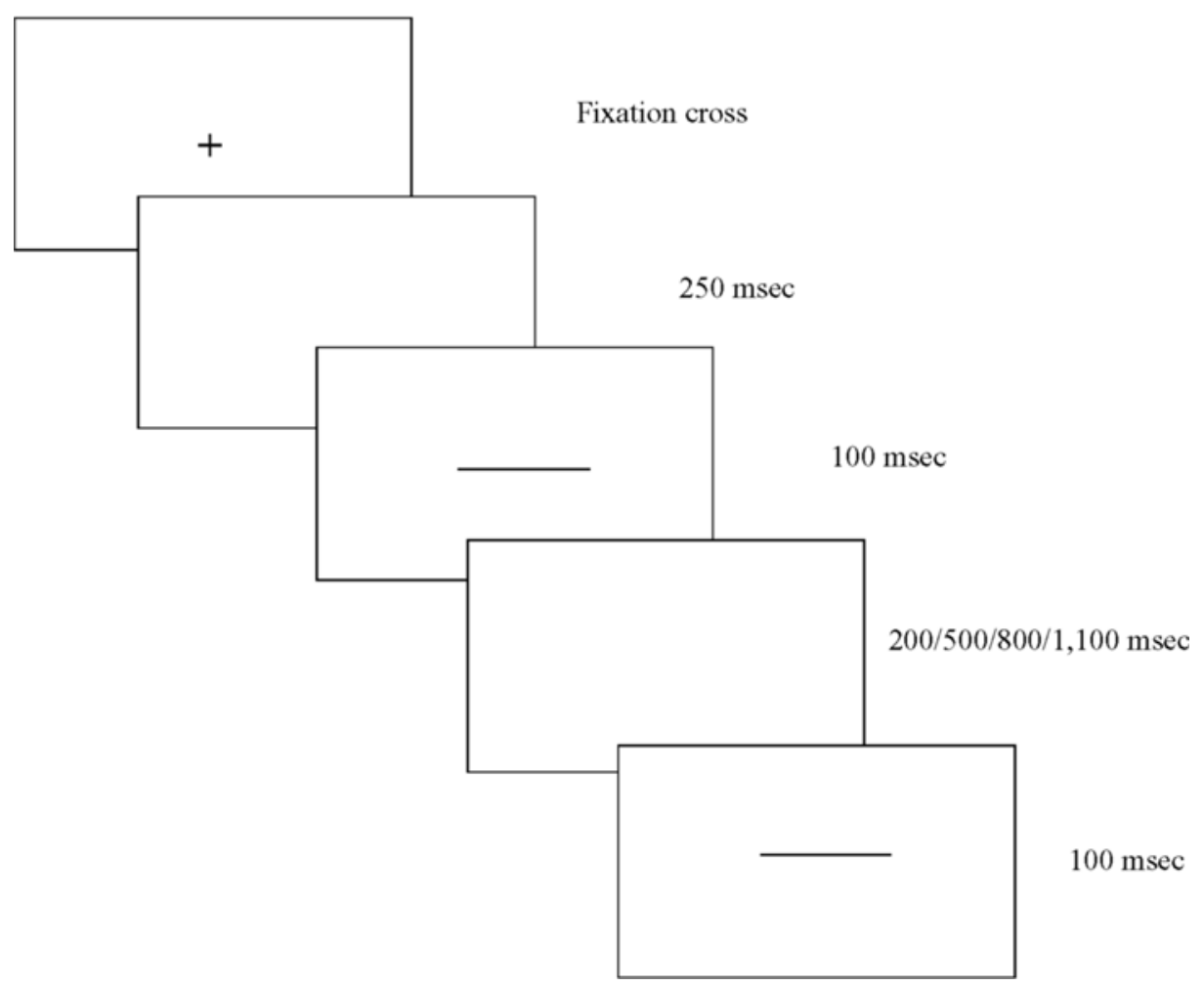

Figure 1. The sequence of displays used on a valid trial. Valid and invalid trials occurred equally often. Participants were required to discriminate only whether the target line was long or short.

proportion of trials presented in each SOA condition. In the unbiased SOA condition, there were equal proportions (.250) of trials in each of the four SOA conditions. In the biased SOA condition, the proportion of trials in the 300 -msec SOA condition was .625, while the proportion of trials in each of the other three SOA conditions was .125.

These proportions were established within each block of 16 trials in the experiment as follows. In the unbiased SOA condition, there were two trials in each of the conditions defined by the factorial combination of validity (valid/invalid) and SOA (300/600/900/ $1,200 \mathrm{msec}$ ) variables. In the biased SOA condition, there were five valid and five invalid trials at the 300 -msec SOA, and one valid and one invalid trial at each of the remaining three SOAs. The presentation of these trial types was randomized within each block.

\section{Results}

RTs on trials that were responded to correctly in each condition were submitted to an outlier analysis to avoid inclusion of suspiciously large or small RTs in further analyses. The modified recursive procedure of Van Selst and Jolicœur (1994) chooses a criterion for exclusion that depends on sample size to ensure that more observations are not systematically eliminated from conditions with fewer observations. Use of this procedure resulted in the elimination of $3.7 \%$ of the observations from subsequent analyses. Mean correct RTs in each cell were then computed using the remaining observations. Mean RTs and error percentages were submitted to fourway analyses of variance (ANOVAs) with validity (valid/ invalid) and SOA (300/600/900/1,200 msec) treated as within-subjects variables and discrimination difficulty (easy/difficult) and SOA bias (unbiased/biased) treated as between-subjects variables. Mean RTs and error percentages for each condition, collapsed across participants, are displayed in Table 1.

In the analysis of RTs, there was a significantinteraction between SOA and validity $\left[F(3,114)=11.70, M S_{\mathrm{e}}=\right.$ $1,728.76, p<.001]$. Subsequent comparisons revealed no significant difference between valid and invalid conditions at the 300-msec SOA $(p>.20)$, but did reveal significant differences between valid and invalid conditions at the 600-, 900-, and 1,200-SOAs $[t(19)=5.08$, $t(19)=7.13, t(19)=3.19$, respectively, all $p \mathrm{~s}<.005]$. For all three of these longer SOAs, responses were slower in the valid condition than in the invalid condition.

There were several other significant effects in the analysis that were of less importance to our research aims. In particular, the main effect of SOA was significant $\left[F(3,228)=6.82, M S_{\mathrm{e}}=2,819.03, p<.001\right]$. Responses were slowest at the $300-\mathrm{msec}$ SOA $(586 \mathrm{msec})$ and fastest at the 900-msec SOA (560 msec). There was also a significant main effect of discrimination difficulty $\left[F(1,76)=27.68, M S_{\mathrm{e}}=55,658.14, p<.001\right]$, with faster responses in the easy discrimination condition than in the difficult discrimination condition. The only other significant effect in this analysis was the interaction be- 
Table 1

Mean Correct Response Times (RTs, in Milliseconds) and Mean Error Percentages (PE) for Target Responses in the Line Identification Task of Experiment 1

\begin{tabular}{|c|c|c|c|c|c|c|c|c|}
\hline \multirow[b]{3}{*}{ Validity } & \multicolumn{8}{|c|}{ SOA (msec) } \\
\hline & \multicolumn{2}{|c|}{300} & \multicolumn{2}{|c|}{600} & \multicolumn{2}{|c|}{900} & \multicolumn{2}{|c|}{1,200} \\
\hline & RT & $\mathrm{PE}$ & RT & $\mathrm{PE}$ & RT & $\mathrm{PE}$ & RT & $\mathrm{PE}$ \\
\hline \multicolumn{9}{|c|}{ Easy-Not Biased } \\
\hline Invalid & 525 & 2.0 & 524 & 3.2 & 488 & 1.9 & 505 & 1.3 \\
\hline Valid & 512 & 1.8 & 550 & 1.3 & 526 & 1.3 & 555 & 1.4 \\
\hline Invalid - valid & 13 & 0.2 & -26 & 1.9 & -38 & 0.6 & -50 & -0.1 \\
\hline \multicolumn{9}{|c|}{ Easy-Biased } \\
\hline Invalid & 531 & 2.1 & 508 & 1.8 & 494 & 1.3 & 493 & 2.0 \\
\hline Valid & 524 & 1.3 & 566 & 1.2 & 542 & 2.5 & 548 & 1.2 \\
\hline Invalid - valid & 7 & 0.8 & -58 & 0.6 & -48 & -1.2 & -55 & 0.8 \\
\hline \multicolumn{9}{|c|}{ Difficult-Not Biased } \\
\hline Invalid & 665 & 1.3 & 587 & 1.1 & 593 & 1.2 & 587 & 1.7 \\
\hline Valid & 657 & 2.6 & 617 & 1.9 & 616 & 1.6 & 618 & 1.6 \\
\hline Invalid - valid & 8 & -1.3 & -20 & -0.8 & -23 & -0.4 & -31 & 0.1 \\
\hline \multicolumn{9}{|c|}{ Difficult-Biased } \\
\hline Invalid & 637 & 2.1 & 604 & 0.7 & 600 & 2.5 & 607 & 1.8 \\
\hline Valid & 643 & 3.9 & 647 & 2.0 & 628 & 2.7 & 646 & 1.5 \\
\hline Invalid - valid & -6 & -1.8 & -43 & -1.3 & -28 & -0.2 & -39 & 0.3 \\
\hline \multicolumn{9}{|c|}{ Overall } \\
\hline Invalid & 590 & 1.9 & 558 & 1.7 & 544 & 1.8 & 548 & 1.7 \\
\hline Valid & 584 & 2.4 & 595 & 1.6 & 578 & 2.0 & 592 & 1.5 \\
\hline Invalid - valid & 6 & -0.5 & -37 & 0.1 & -35 & -0.2 & -44 & 0.2 \\
\hline
\end{tabular}

Note-The four groups differed both in whether the line identification task was easy or difficult and in whether the proportion of trials in each stimulus onset asynchrony (SOA) condition was biased or not biased to favor the shortest SOA.

tween discrimination difficulty and SOA $[F(3,228)=$ $\left.6.08, M S_{\mathrm{e}}=2,819.03, p<.001\right]$. The discrimination difficulty effects were $127,80,97$, and $89 \mathrm{msec}$ in the $300-$, 600-, 900-, and 1,200-msec SOA conditions, respectively, with faster responses in the easy discrimination condition in each case. This pattern of results, together with the main effect of SOA just described, suggests that the effect of discrimination difficulty was greatest at the SOA at which participants were least prepared for onset of the target stimulus (i.e., the shortest SOA). Finally, the three-way interaction between discrimination difficulty, SOA bias, and SOA approached significance $(p<.10)$. This effect appears to be due to a relatively small shift as a result of the SOA bias manipulation in the size of the two-way interaction just described.

There were no significant main effects or interactions in the analysis of error rates.

\section{Discussion}

When the cue-target SOA was longer than $300 \mathrm{msec}$, line length identification responses were reliably slower when the target was of the same length as the cue than when the target was of a different length than the cue. In contrast, in the 300-msec SOA condition, RTs were not significantly different for valid and invalid trials. A similar interaction between validity and SOA is often observed in studies of exogenous spatial orienting (Klein \&
Pontefract, 1994; Maylor, 1985; Mondor, Breau, \& Milliken, 1998; Posner \& Cohen, 1984; Rafal et al., 1989). The similar patterns of results here and in studies of spatial orienting are consistent with the view that similar processes are involved, and in particular with the proposal that the processes responsible for inhibition of return are not specific to the domain of space.

\section{EXPERIMENT 2}

Performance in exogenous spatial orienting studies is often more efficient for valid than for invalid trials at cue-target SOAs less than $300 \mathrm{msec}$ (but see Collie, Maruff, Yucel, Danckert, \& Currie, 2000, and the General Discussion section for critiques of this observation). Indeed, according to the disengage account of inhibition of return described here, one ought to be able to find evidence that attention was engaged on the cue (i.e., a facilitation effect) prior to the point at which inhibition of return is measured. Given that the shortest SOA tested in Experiment 1 was $300 \mathrm{msec}$, it is perhaps not surprising that a facilitation effect was not observed. Yet, a demonstration of this type of facilitation effect would provide added support for the proposal that spatial and nonspatial cuing effects have the same cause.

Initially, we were reluctant to use an SOA shorter than $300 \mathrm{msec}$ because we assumed that it would introduce a 
source of influence on performance that is usually not present in spatial orienting studies. Specifically, if a horizontal cue line were to be followed by a horizontal target line, with both lines centered in the same position on the viewing monitor, a very short SOA might make it difficult for participants to detect that two separate events have occurred, resulting in long RTs on valid trials. We assumed that any attempt to measure cuing effects in our line length identification task using very short cue-target SOAs would have to address this possibility. We did so in this experiment by including two groups of participants. For one group, both the cue and target lines were presented horizontally, as in Experiment 1, while for the other group one of the two lines was horizontal and the other was vertical. The question of interest was whether a facilitation effect would occur with a cue-target SOA less than $300 \mathrm{msec}$. We suspected that such an effect would occur, but perhaps only when the cue and target lines were perpendicular.

\section{Method}

Participants. Forty undergraduate students with a mean age of 22 years participated for course credit. Participants were recruited from an introductory psychology course and a 2nd-year course in cognition at McMaster University. Twenty participants were assigned randomly to each of two groups. All participants reported normal or corrected-to-normal visual acuity.

Apparatus and Stimuli. The apparatus and stimuli in this experiment were the same as in Experiment 1 with the following exceptions. The short and long lines subtended $3.5^{\circ}$ and $10.8^{\circ}$ in length, respectively. This ratio of slightly more than $1: 3$ in line lengths lies between that for the easy and difficult conditions in Experiment 1 , but relatively close to the 1:3.9 ratio for the easy condition. For the collinear group, the lines were otherwise identical to those in Experiment 1. For the perpendicular group, one of the two lines was vertical while the other was horizontal. Both the horizontal and vertical lines were centered on the location occupied by the fixation point.

Procedure and Design. The procedure and design of this experiment were identical to that in Experiment 1 with the following exceptions. The SOAs in this experiment were 157, 457, 757, and $1,057 \mathrm{msec}$. Note that the difference between adjacent SOAs is $300 \mathrm{msec}$, as was the case in Experiment $1(300,600,900,1,200 \mathrm{msec})$; each SOA in this experiment was simply $143 \mathrm{msec}$ less than the corresponding SOA in Experiment 1. The shorter SOAs in this experiment were produced by shortening the duration of the cue by $43 \mathrm{msec}(100-57 \mathrm{msec})$, and each of the four cue-target interstimulus intervals by $100 \mathrm{msec}$.

For both groups, the experiment again consisted of 1 block of 16 practice trials followed by 20 blocks of 16 experimental trials. In the collinear group, both the cue and target lines were presented horizontally, as in Experiment 1. Each block contained 2 trials in each of the eight conditions defined by factorially combining the SOA (four levels) and validity (two levels) variables. In the perpendicular group, if the cue was presented horizontally, then the target was presented vertically, and vice versa. Thus, each of the eight conditions defined by factorially combining the SOA and validity variables was presented once with a horizontal cue and once with a vertical cue in each block.

\section{Results}

Correct RTs were submitted to the outlier analysis used in Experiment 1 (Van Selst \& Jolicœur, 1994), which resulted in the elimination of $3.1 \%$ of the observations from subsequent analyses. Mean correct RTs in each cell were then computed using the remaining observations. Mean RTs and error percentages were submitted to three-way ANOVAs with validity (valid/invalid) and SOA $(157 / 457 / 757 / 1,057 \mathrm{msec})$ treated as within-subjects variables and orientation (collinear/perpendicular) treated as a between-subjects variable. Mean RTs and error percentages for each condition, collapsed across participants, are displayed in Table 2 .

Response times. In the analysis of RTs, there was a significant interaction between SOA and validity $[F(3,114)=$ $\left.18.78, M S_{\mathrm{e}}=541.63, p<.001\right]$. Subsequent analyses of the validity effect at each SOA were then conducted. For the shortest SOA $(157 \mathrm{msec})$, responses were significantly faster for valid than for invalid trials $[t(39)=3.61$, $p<.001]$. In contrast, for the three longer SOAs $(457$,

Table 2

Mean Correct Response Times (RTs, in Milliseconds) and Mean Error Percentages (PE) for Target Responses in the Line Length Identification Task of Experiment 2

\begin{tabular}{|c|c|c|c|c|c|c|c|c|}
\hline \multirow[b]{3}{*}{ Validity } & \multicolumn{8}{|c|}{ SOA $(\mathrm{msec})$} \\
\hline & \multicolumn{2}{|c|}{157} & \multicolumn{2}{|c|}{457} & \multicolumn{2}{|c|}{757} & \multicolumn{2}{|c|}{1,057} \\
\hline & RT & $\mathrm{PE}$ & RT & PE & RT & $\mathrm{PE}$ & $\mathrm{RT}$ & $\mathrm{PE}$ \\
\hline \multicolumn{9}{|c|}{ Perpendicular } \\
\hline Invalid & 548 & 3.4 & 478 & 2.1 & 447 & 2.4 & 450 & 2.2 \\
\hline Valid & 523 & 1.1 & 498 & 2.0 & 471 & 1.5 & 471 & 0.6 \\
\hline Invalid - valid & 25 & 2.3 & -20 & 0.1 & -24 & 0.9 & -21 & 1.6 \\
\hline \multicolumn{9}{|c|}{ Collinear } \\
\hline Invalid & 566 & 2.1 & 482 & 1.4 & 466 & 2.0 & 468 & 1.6 \\
\hline Valid & 541 & 0.6 & 508 & 0.8 & 479 & 1.6 & 481 & 1.9 \\
\hline Invalid - valid & 25 & 1.5 & -26 & 0.6 & -13 & 0.4 & -13 & -0.3 \\
\hline \multicolumn{9}{|c|}{ Overall } \\
\hline Invalid & 557 & 2.8 & 480 & 1.8 & 457 & 2.2 & 459 & 1.9 \\
\hline Valid & 532 & 1.9 & 503 & 1.4 & 475 & 1.6 & 476 & 1.3 \\
\hline Invalid - valid & 25 & 0.9 & -23 & 0.4 & -18 & 0.6 & -17 & 0.6 \\
\hline
\end{tabular}

Note-SOA, stimulus onset asynchrony. 
$757,1,057 \mathrm{msec})$, responses were significantly slower for valid than for invalid trials $[t(39)=3.95, t(39)=3.76$, $t(39)=3.29$, respectively, all $p \mathrm{~s}<.005]$.

There were two other significant effects in the analysis that were of less theoretical interest. The main effect of SOA was significant $\left[F(3,114)=80.08, M S_{\mathrm{e}}=\right.$ $1,345.50, p<.001]$. Responses were slowest at the $157-\mathrm{msec}$ SOA $(545 \mathrm{msec})$ and fastest at the $757-\mathrm{msec}$ SOA (466 msec), generally decreasing with increasing SOA. Also, the main effect of validity was significant $\left[F(1,38)=5.22, M S_{\mathrm{e}}=1,108.29, p<.05\right]$. Overall, responses were slower for valid than for invalid trials (497 vs. $488 \mathrm{msec}$ ).

Errors. In the analysis of error percentages, the only significant effect was the main effect of validity, with more errors on invalid trials than on valid trials. This pattern of results was particularly pronounced for the $157-\mathrm{msec}$ SOA condition $(2.8 \%$ vs. $0.9 \%)[t(39)=2.51, p<.05]$, which is in accord with the pattern of RTs. A separate analysis of the error percentages for the three longer SOA conditions revealed no significant difference $(p>.10)$.

Comparison of Experiments 1 and 2. To compare the validity effects observed here with those of Experiment 1, mean RTs from this experiment were submitted to an ANOVA that treated experiment as a between-subjects variable and validity and SOA as within-subjects variables. For the purpose of this analysis, differences in the SOAs used in the two experiments were ignored, and SOA levels can be thought of as very short, short, long, and very long, relative to accompanying SOAs in the same experiment. Only the data from the easy discrimination task of Experiment 1 were analyzed, leaving data from 40 participants for both Experiments 1 and 2 .

As expected, this analysis revealed a significant interaction between validity and SOA $[F(3,234)=18.89$, $\left.M S_{\mathrm{e}}=1,363.42, p<.001\right]$. However, the results of most interest were interactions that involved the experiment factor. If the absence of a facilitation effect at the shortest SOA in Experiment 1 were simply due to the shortest SOA not being short enough, then one would expect a more positive cuing effect for the shortest SOA in Experiment 2 than for the shortest SOA in Experiment 1. However, at longer SOAs, this difference in cuing effects between experiments ought to disappear. Together, these patterns of data would produce a significant interaction between experiment, validity, and SOA. In fact, this interaction did not approach significance $(F<1)$. Rather, there was a significant interaction between experiment and validity $\left[F(1,78)=8.94, M S_{\mathrm{e}}=2,475.42, p<.005\right]$. As can be seen in Figure 2, the cuing effects in Experiment 2 were more positive than in Experiment 1 across the range of SOAs.

Of less relevance, there was a significant interaction between experiment and SOA $\left[F(3,234)=24.32, M S_{\mathrm{e}}=\right.$ $2131.48, p<.001]$. Whereas RTs were almost identical for the shortest and longest SOA conditions in Experiment 1 ( 523 vs. $525 \mathrm{msec}$ ), RTs were much higher in the shortest SOA condition than in the longest SOA condition in Experiment 2 (546 vs. $468 \mathrm{msec}$ ).

\section{Discussion}

The pattern of results in this experiment was similar to that observed in exogenous spatial orienting studies. Responses were faster for valid than for invalid trials at the shortest cue-target SOA, while the opposite result was observed for the three longer SOAs. These results are consistent with the view that spatial and nonspatial cuing effects have the same cause.

At the same time, two findings were not anticipated. First, the pattern of cuing effects was identical for the collinear and perpendicular groups. Thus, our concern that collinear cues and targets would be particularly difficult to distinguish as separate events appears to have been unwarranted. Second, when Experiments 1 and 2

\section{Cuing Effects in the \\ Line Length Identification Task}

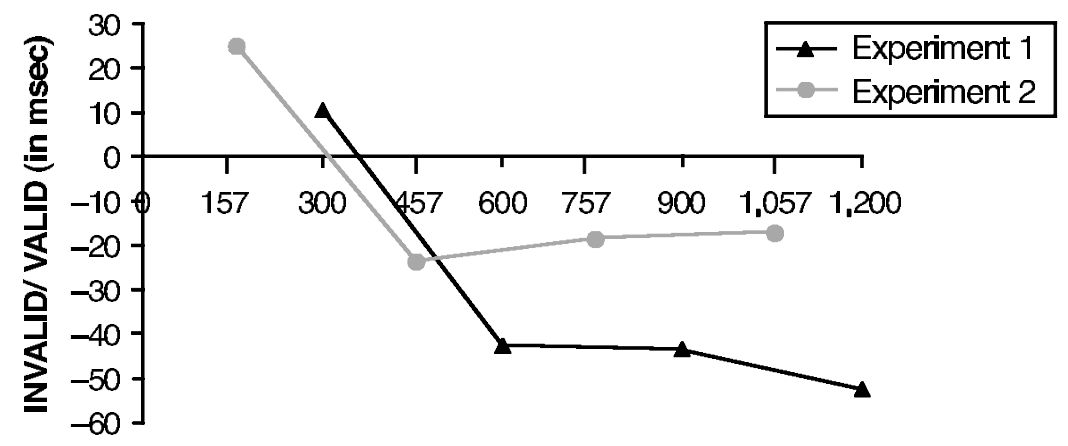

\section{SOA (in msec)}

Figure 2. Response time cuing effects in the line length identification task in Experiments 1 and 2 . 
were considered together, the results revealed more positive cuing effects in Experiment 1 at all SOAs rather than solely at the shortest SOA. This pattern of results is difficult to reconcile with a disengage account of inhibition of return, because it is not clear why disengaging attention from a cue that produces a small facilitation effect would produce a large inhibition of return effect.

Instead, the results seem more compatible with an opponent process approach to explaining cuing effects (see also Danziger \& Kingstone, 1999; Klein, 2000; Mondor, 1999; Tipper et al., 1997). According to this view, cuing effects at any particular SOA reflect the joint influence of two processes, one that speeds performance for valid relative to invalid trials (a positive influence), and another that slows performance for valid relative to invalid trials (a negative influence). An opponent process account can explain the results of Experiments 1 and 2 in a relatively parsimonious manner. Specifically, the procedural changes made from Experiment 1 to Experiment 2, which included a change in cue duration from 100 to $57 \mathrm{msec}$, appear to have affected the negative influence on performance while leaving the positive influence on performance unaffected. Thus, although a facilitation effect was observed in Experiment 2 and not in Experiment 1, the process responsible for facilitating performance for valid relative to invalid trials at short SOAs may have contributed to performance equally in the two experiments. Further evidence for this opponent process view of cuing effects is provided in Experiment 3.

\section{EXPERIMENT 3}

The results in Experiments 1 and 2 were similar to those observed in exogenous spatial orienting studies. However, given that the task required a line length decision, and that validity was defined by cue and target line length, performance differences for valid and invalid targets could be the product of response biases. In other words, a simple bias to respond "long" following short cues and to respond "short" following long cues would produce slower responses for valid than for invalid trials. Note that a response bias of this sort cannot explain inhibition of return effects in many spatial orienting studies, because the task in spatial orienting studies often requires detection of target onset. A target detection task rules out a simple response bias account because re- sponse selection is orthogonal to the dimension used to define validity. To address this issue in our study, participants in Experiment 3 were presented the same stimuli as in Experiment 1, but were required to detect the onset of the target line.

\section{Method}

Participants. Twenty undergraduate students with a mean age of 20 years participated in the experiment for course credit. Participants were recruited from an introductory psychology course at McMaster University. All participants reported normal or corrected-to-normal visual acuity.

Apparatus and Stimuli. The apparatus and stimuli used in this experiment were identical to those used in Experiment 1. The lengths of the lines subtended $1.75^{\circ}$ and $6.75^{\circ}$ of visual angle, which corresponds to those used in the easy discrimination condition of Experiment 1.

Procedure. The procedure of this experiment was identical to that of Experiment 1 with the following exceptions. Participants were required to detect the onset of the target line rather than to identify whether it was short or long. Participants initiated each trial by pressing "b" on the keyboard and recorded their response on target trials by pressing the space bar on the keyboard upon target onset. On $20 \%$ of the trials no target appeared, and participants were required to withhold a response. With the addition of these catch trials, there were 20 blocks of 20 trials each, with 4 catch trials per block. On the catch trials, a 2,000-msec blank interval followed the offset of the cue, which was then followed by the fixation cross for the next trial.

\section{Results}

Correct RTs on target trials were submitted to the same outlier elimination procedure as in Experiments 1 and 2 (Van Selst \& Jolicœur, 1994). This outlier procedure eliminated $2.9 \%$ of the data from further analyses. Mean RTs for each condition were computed using the remaining observations and were then submitted to a repeated measures ANOVA that treated validity (valid/invalid) and SOA (300/600/900/1,200 msec) as within-subjects factors. Mean RTs for each condition, collapsed across participants, are displayed in Table 3.

There was a main effect of validity $[F(1,19)=16.35$, $\left.M S_{\mathrm{e}}=1,586.36, p<.001\right]$. Responses were slower for valid trials ( $387 \mathrm{msec})$ than for invalid trials $(362 \mathrm{msec}$ ).

The interaction between validity and SOA approached significance $\left[F(3,57)=2.56, M S_{\mathrm{e}}=1,073.72, p<.07\right]$. Interestingly, the source of this interaction appears to be a particularly large negative validity effect (slower responses for valid trials) at the shortest SOA. This inter-

Table 3

Mean Correct Response Times (RTs, in Milliseconds) and Mean Error Percentage (PE) for Target Responses in the Line Length Detection Task of Experiment 3

\begin{tabular}{|c|c|c|c|c|c|c|c|c|}
\hline \multirow[b]{3}{*}{ Validity } & \multicolumn{8}{|c|}{$\mathrm{SOA}(\mathrm{msec})$} \\
\hline & \multicolumn{2}{|c|}{300} & \multicolumn{2}{|c|}{600} & \multicolumn{2}{|c|}{900} & \multicolumn{2}{|c|}{1,200} \\
\hline & RT & PE & RT & PE & $\mathrm{RT}$ & PE & $\mathrm{RT}$ & $\mathrm{PE}$ \\
\hline Invalid & 379 & 1.7 & 354 & 2.1 & 351 & 1.5 & 365 & 1.1 \\
\hline Valid & 429 & 3.4 & 372 & 1.7 & 371 & 0.5 & 379 & 2.2 \\
\hline Invalid - valid & -50 & -1.7 & -18 & 0.4 & -20 & 1.0 & -14 & -1.0 \\
\hline
\end{tabular}

Note-SOA, stimulus onset asynchrony. 
pretation was supported by an ANOVA that included only the three longest SOAs. In this analysis, the validity by SOA interaction did not approach significance $(F<1)$, while the main effect of validity remained significant $\left[F(1,19)=9.68, M S_{\mathrm{e}}=924.05, p<.01\right]$. The only other significant effect in the overall analysis was the main effect of SOA $\left[F(3,57)=10.32, M S_{\mathrm{e}}=1540.45\right.$, $p<.001]$. Responses were particularly slow at the shortest SOA (Table 3).

An analysis of target misses (errors in which a target appeared but no response was made; see Table 2) that treated SOA and validity as within-subjects variables was also conducted. This analysis revealed a significant main effect of SOA $\left[F(3,57)=3.16, M S_{\mathrm{e}}=5.15, p<.05\right]$, with the highest number of errors occurring in the shortest SOA condition. No other effect was significant in this analysis. False alarms (errors in which a target did not appear but a response was made) were infrequent, occurring on $3 \%$ of catch trials. Anticipation errors (errors in which a response was made prior to the onset of a target) were also infrequent, occurring on $2 \%$ of target trials.

\section{Discussion}

Two results are of importance in this experiment. First, responses were slower for valid than for invalid targets. Because a detection task was used, this result cannot be attributed to a simple bias favoring the response opposite that elicited by the cue. In this sense, the present data again support the view that inhibition of return is not restricted to the dimension of space. Second, however, the interaction between validity and SOA that often occurs in exogenous spatial cuing tasks, and that occurred for the line length identification task in Experiments 1 and 2, was not observed in Experiment 3. Instead, negative validity effects were observed at all SOAs, with the largest effect occurring at the shortest SOA. If a fixed time course of cuing effects-with facilitation at short SOAs and the opposite effect at long SOAs-is used as a criterion for judging whether spatial and nonspatial cuing effects have a common cause, then the results of this experiment contradict the common cause hypothesis.

On the other hand, the opponent process framework assumes that the time course of cuing effects is not fixed, and that it depends on the relative contributions of two processes across the range of SOAs tested. From this perspective, the cuing effects in this experiment could well reflect the same process that produced negative validity effects in the prior experiments, but without the opposing process that speeds performance for valid relative to invalid trials at short cue-target SOAs. This proposal is addressed in more detail in the General Discussion section.

\section{EXPERIMENT 4}

In Experiments 1 and 2, a two-alternative forced-choice discrimination task was used, and negative validity effects emerged only at longer cue-target SOAs. In con- trast, in Experiment 3 a detection task was used, and negative validity effects were observed across the range of cue-target SOAs. These results raise the possibility that the time course of nonspatial cuing effects depends entirely on whether a detection task or a two-alternative forced-choice discrimination task is used. In accord with this proposal, Taylor and Klein (1998a; see also Fox \& DeFockert, 2001) reported negative validity effects at all SOAs in a detection task with validity defined by target color. What is not known is whether the color cuing effects measured by Taylor and Klein (1998a) are sensitive to the nature of the task in the same manner as the line length cuing effects observed in Experiments 1-3. To examine this issue, we conducted an experiment that was identical to Experiment 1, but that used valid and invalid color targets. If line length and color cuing effects respond similarly to changes in task, then color cuing effects in this experiment ought to be dissimilar to those reported by Taylor and Klein (1998a) and similar to those observed in Experiment 1, with negative validity effects emerging only at longer cue-target SOAs.

\section{Method}

Participants. Eighteen undergraduate students with a mean age of 20 years participated in the experiment for course credit. Participants were recruited from an introductory psychology course at McMaster University. All participants reported normal or corrected-to-normal visual acuity.

Apparatus and Stimuli. The apparatus and stimuli in this experiment were identical to those used in Experiment 1 with the exception that the cue and target were colored rectangles displayed centrally, rather than line segments presented centrally. The colored rectangles subtended $3.4^{\circ}$ horizontally and $0.7^{\circ}$ vertically.

Procedure. The procedure of this experiment was identical to that of Experiment 1 except that participants identified the color of a target rectangle rather than the length of a target line. Although four colors were used in the experiment (red, green, blue, yellow), only two colored rectangles were used for any given participant. Each of the six combinations of two colors selected from four (i.e., blue-red, blue-green, blue-yellow, red-green, red-yellow, greenyellow) was used for 3 participants. Participants pressed the " $x$ " key on the keyboard for one target color and the " $\mathrm{m}$ " key on the keyboard for the other target color. The timing parameters of each trial were identical to those in Experiment 1, as was the number of trials per block and the overall number of trials in the experiment. Valid trials were those in which the colors of the cue and target were the same and invalid trials were those in which the colors of the cue and target were different.

\section{Results}

Correct RTs were submitted to the same outlier elimination procedure as in previous experiments (Van Selst \& Jolicœur, 1994), which eliminated $2.6 \%$ of the data from further analyses. Mean RTs in each condition were computed using the remaining observations, and were then submitted to a repeated measures ANOVA that treated validity (valid/invalid) and SOA (300/600/900/ $1,200 \mathrm{msec}$ ) as within-subjects factors. Mean RTs for each condition, collapsed across participants, are displayed in Table 4. 
Table 4

Mean Correct Response Times (RTs, in Milliseconds) and Mean Error Percentage (PE) for Target Responses in the Color Identification Task of Experiment 4

\begin{tabular}{|c|c|c|c|c|c|c|c|c|}
\hline \multirow[b]{3}{*}{ Validity } & \multicolumn{8}{|c|}{ SOA (msec) } \\
\hline & \multicolumn{2}{|c|}{300} & \multicolumn{2}{|c|}{600} & \multicolumn{2}{|c|}{900} & \multicolumn{2}{|c|}{1,200} \\
\hline & $\mathrm{RT}$ & $\mathrm{PE}$ & $\mathrm{RT}$ & $\mathrm{PE}$ & $\mathrm{RT}$ & $\mathrm{PE}$ & $\mathrm{RT}$ & $\mathrm{PE}$ \\
\hline Invalid & 555 & 1.7 & 512 & 1.4 & 485 & 1.6 & 471 & $\overline{2.2}$ \\
\hline Valid & 579 & 3.2 & 515 & 1.0 & 490 & 1.4 & 474 & 1.5 \\
\hline Invalid - valid & -24 & -1.5 & -3 & 0.4 & -5 & 0.2 & -3 & 0.7 \\
\hline
\end{tabular}

Note-SOA, stimulus onset asynchrony.

There was a significant main effect of validity $\left[F(1,17)=6.71, M S_{\mathrm{e}}=401.88, p<.05\right]$, with slower responses for valid $(515 \mathrm{msec})$ than for invalid $(505 \mathrm{msec})$ trials. There was no significant interaction between validity and SOA $(p>.20)$. Given that the largest negative validity effect occurred for the shortest SOA condition, it seems clear that the pattern of validity effects resembled that from Experiment 3 more than that from Experiments 1 and 2 . The only other significant effect in the analysis was the main effect of SOA $[F(3,51)=42.10$, $\left.M S_{\mathrm{e}}=1,461.72, p<.001\right]$. Responses were slowest at the shortest SOA and fastest at the longest SOA.

\section{Discussion}

The time course of cuing effects in this experiment was clearly different from that observed in Experiment 1, although a two-alternative forced-choice task was used in both experiments. Further, the time course of cuing effects in this experiment was similar to that observed in Experiment 3 (see also Fox \& DeFockert, 2001; Taylor \& Klein, 1998b), although a two-alternative forced-choice task was used in the present experiment and a detection task was used in Experiment 3. These results indicate that the time course of nonspatial cuing effects is determined by both the nature of the task and the stimulus attribute that defines validity.

\section{GENERAL DISCUSSION}

With few exceptions, inhibition of return has been studied using a spatial cuing method. The present study examined whether inhibition of return effects can occur using a nonspatial cuing method. In the line length detection task of Experiment 3, responses were slower for valid than for invalid trials at all SOAs and, if anything, this effect was largest at the shortest SOA. This result is similar to that reported in two prior color cuing studies that used a detection task (Taylor \& Klein, 1998a; see also Fox $\&$ DeFockert, 2001). Given that the time course of these nonspatial cuing effects differs from that often observed in spatial cuing studies, these results might be taken as support for the view that different processes are responsible for cuing effects in spatial and nonspatial tasks.

However, a different pattern of results was observed in Experiments 1 and 2. In these experiments, partici- pants responded by indicating the length of the target line rather than by detecting its onset. With this task, responses were slower for valid than for invalid targets only when the cue-target SOA was longer than $300 \mathrm{msec}$. The stimuli presented to participants in Experiments 1 and 3 were identical, and yet the pattern of cuing effects was very different. Clearly, in a cuing task in which validity is defined by line length, the time course of cuing effects is not stable. Rather, it varies as a function of task.

That the time course of cuing effects changed as a function of task across Experiments 1-3 calls into question whether this property of performance can be used to evaluate the relation between spatial and nonspatial cuing effects (Taylor \& Klein, 1998a). Stated differently, it is impossible to determine whether "the" time course of cuing effects for the nonspatial property of line length matches that for spatial location, since the property of line length does not produce a single, stable time course that can be used for comparison. Furthermore, a close look at the literature reveals that the time course of spatial cuing effects is also sensitive to a variety of task variables. For example, in several spatial cuing studies, Lupiáñez, Milan, Tornay, Madrid, and Tudela (1997; Lupiáñez et al., 2001) have found that inhibition of return emerges at longer SOAs in forced-choice discrimination tasks than in detection tasks. In other spatial cuing studies, researchers have reported slower responses for valid targets than for invalid targets even at very short cue-target SOAs (Bennett \& Pratt, 2001; Tassinari, Aglioti, Chelazzi, Peru, \& Berlucchi, 1994). Together, these studies and others demonstrate that the time course widely thought to be characteristic of exogenous spatial cuing effects is not always observed (see Collie et al., 2000 , for an overview).

This variability in the time course of cuing effects in both spatial and nonspatial cuing tasks leads us to conclude that some of the inferences made about nonspatial cuing effects in prior studies may not be justified. In particular, the absence of a facilitation effect at short cue-target SOAs does not imply that negative validity effects in nonspatial cuing tasks have a different cause than inhibition of return effects in spatial cuing tasks (Fox \& DeFockert, 2001; Taylor \& Klein, 1998a).

Of the two theoretical accounts for inhibition of return effects offered in the introduction, variability in the time course of cuing effects is more easily accommodated by 
the opponent process account than by the disengage account. According to the disengage account, inhibition of return effects are a direct by-product of attending to a location and then disengaging attention from that location. As such, inhibition of return effects ought to appear only after attention has been disengaged, and should always be preceded by a facilitation effect at shorter cue-target SOAs. Clearly, a strong version of this hypothesis cannot account for the type of variability in time course of cuing effects observed in the present study.

In contrast, according to the opponent process account, cuing effects always measure the joint contribution to performance of two processes, one that slows performance on valid relative to invalid trials and another that speeds performance on valid relative to invalid trials. Inhibition of return effects then occur when the former process outweighs the latter (see also Danziger \& Kingstone, 1999; Klein, 2000; Mondor, 1999; Tipper et al., 1997). This hypothesis can accommodate substantial variability in the time course of cuing effects by assuming that the two processes are independent and can affect performance to varying degrees.

For example, the process that speeds performance for valid relative to invalid trials may or may not contribute to performance at short cue-target SOAs. If this facilitatory process does not contribute to performance, then inhibition of return effects may be observed at all SOAs. In contrast, if the facilitatory process contributes to performance at short SOAs but not at long SOAs, then inhibition of return effects might emerge only at longer SOAs. From this standpoint, the data from the present study could be accounted for by assuming that there was a facilitatory process to offset the process responsible for inhibition of return effects in the line length identification tasks of Experiments 1 and 2 but not in the detection task of Experiment 3.

Similarly, the process that slows performance for valid relative to invalid trials can contribute to performance to varying degrees. If the contribution to performance of this interference process is large relative to the facilitation process, then a facilitation effect might not be observed at short SOAs. In contrast, if the contribution to performance of the interference process is small relative to the facilitation process, then a significant facilitation effect ought to occur at short SOAs. In this way, changes in the strength of the interference process could explain the different results in Experiments 1 and 2 (Figure 2). As noted, we conjecture that it may have been changes in the cue duration that altered the contribution of the interference process to performance.

In summary, changes in cue duration from Experiment 1 to Experiment 2 appear to have affected one component process contributing to cuing effects, and changes in task from Experiments 1 and 2 to Experiment 3 appear to have affected a different component process contributing to cuing effects. Together, the results suggest that both task and stimulus factors can introduce processing variability that alters the time course of cuing effects.
Although this opponent process hypothesis provides a reasonable post hoc description of performance in the present study, to be useful as a tool to predict variability in cuing effects, some specification of the component processes of cuing effects is required. A brief specification of opponent processes that could contribute to both spatial and nonspatial cuing effects is addressed in the following two sections.

\section{The Facilitation Component of Cuing Effects}

At the outset, it should be acknowledged that the processes underlying the facilitation effects in our line length cuing task may differ from those underlying the facilitation effect in spatial cuing tasks. To provide a concrete example, the facilitation component of performance in Experiments 1 and 2, in which the task was line length identification, could well have been caused by a response biasing influence of the cue (i.e., response priming). This hypothesis has some support in our study, since the facilitation component was absent in Experiment 3 when the task was changed to target detection. In contrast, response bias/priming cannot explain facilitation effects that occur at short cue-target SOAs in spatial orienting studies that use a detection task. Therefore, although the time course of cuing effects in Experiments 1 and 2 is consistent with that observed in spatial cuing studies, the processes that produce the facilitation components of performance may differ.

Nevertheless, we are drawn to the possibility that a single framework might be used to explain the facilitation component of both nonspatial and spatial cuing effects. To account for both spatial and nonspatial cuing effects, the process responsible for facilitation effects at short cue-target SOAs would have to be broadened beyond the spatiocentric notion of automatic capture of attention at the location of the cue. To our knowledge, the only prior specification of this type was offered by Mondor (1999; Mondor \& Lacy, 2001) to explain the time course of auditory cuing effects. Mondor proposed that selection of auditory information is mediated by an attentional template. Under normal conditions, the attentional template is determined by a mix of the preceding auditory stream and the listener's goals. Selection of a current stimulus for further processing is then determined by the similarity between that stimulus and the attentional template, with faster selection for more similar stimuli. To apply this general idea to an exogenous cuing study, Mondor proposed that the attentional template is set in accordance with the properties of the abrupt onset cue. Therefore, targets that match the cue are selected for further processing faster than targets that mismatch the cue, which accounts for the facilitation effect in cuing studies at short cue-target SOAs.

The attentional template proposal of Mondor (1999) broadens the process responsible for facilitation effects in such a way that it explains facilitation effects on the basis of matches between cue and target not only in location, but also in frequency, duration, intensity, and timbre. 
However, the attentional template proposal would not readily accommodate the facilitation effect observed in Experiment 2 of this study. Recall that this facilitation effect depended on task (i.e., it occurred for line length identification in Experiment 2 but not for line length detection in Experiment 3), and it did not depend on the physical similarity between cue and target (i.e., it was of equal size for the perpendicular and collinear groups). Thus, the facilitation effects observed in Experiment 2 might well be attributed to processes outside the domain of the attentional template model, such as response priming/bias (see Mondor \& Lacy, 2001, for a similar discussion). Alternatively, a still broader framework might be proposed that captures both the auditory cuing results of Mondor and colleagues (Mondor, 1999; Mondor, Breau, \& Milliken, 1998; Mondor \& Lacy, 2001) and the results of the present study. Although there is some risk of broadening theoretical discussion beyond the point of utility, we offer the following framework to explain the facilitation component of both spatial and nonspatial cuing effects.

We follow the lead of Mondor (1999) in assuming that the processes that cause cuing effects are related to the streaming of incoming information into meaningful events (Bregman, 1990). Also consistent with the proposal of Mondor, we assume that a cue sets up an internal representation against which a target is compared. However, we define this internal representation broadly as an event representation and assume that it can include both task-relevant properties of the cue and information about how that cue might be linked to action. Responses are faster for targets that match cues when the task allows participants to use similarity between cue and target events to quickly and accurately interpret the status of a matching target.

To appreciate how this framework explains the results of the present study, consider the line length identification task used in Experiment 1. Although an overt response was not required for the cue in this experiment, we assume that participants engage nevertheless in some amount of covert identification of the length of the cue upon its presentation. Consequently, an event representation of the cue includes information about its length (the task-relevant attribute) and the appropriate response to a stimulus of that length. When a target of matching length appears, the similarity between the processes used to encode the cue and target triggers a reinstatement process, whereby the memory representation of the cue event is retrieved and used to respond to the matching target. If response selection using this reinstatement process was particularly efficient in the line length identification tasks of Experiments 1 and 2, this would explain why there appeared to be a facilitation component to cuing effects in these experiments. In contrast, reinstatement of a prior event in which a line of similar length was displayed may provide no useful information in a target detection task, which would account for the absence of a facilitation component to cuing effects in Experiment 3 .
This notion that cuing effects can be driven by the retrieval of prior perceptual events is derived from ideas proposed originally by Kahneman, Treisman, and Gibbs (1992). They suggested that onset of a perceptual event initiates a correspondence process that works backward in time, and that links current perceptual processing with similar object files (i.e., event representations) stored in memory. According to this view, perception of a current event may be speeded by integrating that event with a similar prior event. To the extent that this perceptual integration principle holds, it provides a general means of explaining facilitation effects in exogenous cuing studies, and its breadth does not restrict it to any particular sensory modality or stimulus dimension.

Cast in broader terms still, we propose that a current event can be perceived and categorized nonanalytically by reinstating a memory for how a similar item was perceived and categorized, or it can be perceived and categorized in a more perceptually analytic manner. To the extent that the former process is more efficient than the latter, facilitation effects ought to occur in cuing studies, and the cause of the facilitation effect might be thought of as a building block of automaticity (Jacoby, 1978; Kahneman et al., 1992; Logan, 1988; Pashler \& Baylis, 1991).

\section{The Interference Component of Cuing Effects}

According to the opponent process hypothesis, inhibition of return effects occur when the influence of a process that slows responses to valid trials relative to invalid trials is larger than the influence of the facilitation component just described. From this perspective, disengagement of attention from the cued location does not cause responses to be slower for valid trials, but instead reveals the influence of a second process that slows responses to valid trials. This opponent process logic fits nicely with the perceptual integration notion just introduced. Thus, for short cue-target SOAs, an existing event representation may be retrieved and updated on valid trials, and this process may occur more quickly than a new event representation can be created on invalid trials (Kahneman et al., 1992). For longer cue-target SOAs, one of two processing scenarios may occur.

One possibility is that the longer cue-target SOA disrupts the perceptual integration process, slowing it to the point that updating an existing event representation on valid trials is actually slower than creating a new event representation on invalid trials. Inherent in this view is the notion that perceptual integration processes are automatic. In the context of a cuing study, this automaticity might imply that perceptual integration continues to occur and contribute to performance beyond the SOA at which it usefully contributes to performance on valid trials.

A second possibility is that performance for both valid and invalid trials at longer cue-target SOAs depends on the speed with which new event representations can be created. Inhibition of return effects would then imply that new event representations are encoded more effi- 
ciently for invalid than for valid targets. This idea perhaps fits better with the notion that, at some point following the cue, participants disengage attention from the cue. From this perspective, disengagement from the cue might be thought of as a voluntary decision by the participant to process the target as an event separate from the cue. Whether encoding a valid target as a new perceptual event is slowed by the requirement to differentiate similar cue and target events (Mondor, 1999; see also Milliken, Joordens, Merikle, \& Seiffert, 1998) or by the fact that attentional resources are preferentially allocated to invalid (novel) over valid (familiar) targets (Johnston \& Hawley, 1994; Yantis \& Jonides, 1984) is an interesting problem to be addressed in future work.

\section{Alternative Accounts of the Present Results}

The broad theoretical framework sketched in the preceding two sections represents one approach to explaining the results of the present study, and of course there are others. An alternative to broadening current theoretical accounts might instead focus on details of the line length cuing procedure used here, perhaps looking for a source of influence on performance that is available with this procedure but not with a spatial cuing procedure. For example, when two lines of different lengths are presented one after another in the same spatial location, some form of perceptual illusion, such as apparent motion, may occur. In turn, the apparent motion might be used to make an inference about the length of the target line.

In general, the notion that an additional source of information may be available on invalid trials relative to valid trials is a useful starting point for developing an explanation of negative validity effects, whether in spatial or in nonspatial cuing tasks. However, the case for apparent motion causing negative validity effects in the present study is not particularly strong. Although apparent motion effects are sensitive to a variety of presentation parameters (e.g., the distance separating the two events, the brightness and duration of the two events), they are typically strongest when the temporal interval between events is in the order of $100 \mathrm{msec}$ (Palmer, 1999). Thus, one would expect any influence of apparent motion to be stronger at shorter than at longer cue-target intervals in our experiments, which was the case only in Experiment 3. Furthermore, apparent motion effects are sensitive to properties such as the shape and orientation of the two inducing events (Kolers, 1972). In Experiment 2, the perpendicular and collinear groups differed markedly in terms of the relative orientation of the cues and targets, and yet the cuing effects were nearly identical. Assuming that the correspondence process underlying apparent motion would differ in the collinear and perpendicular groups, the results do not support the notion that apparent motion contributed to the cuing effects.

\section{Summary}

The results from the experiments reported here demonstrate that performance for validly cued targets can be slower than that for invalidly cued targets when validity is defined by the relative lengths of cue and target lines. Just as important, the experiments also produced very different time courses for cuing effects across tasks that used very similar stimuli. We pointed out that the time course of spatial cuing effects is also sensitive to a host of task variables. Since there may be no single, stable time course for cuing effects in either spatial or nonspatial cuing tasks, it may not be possible to use the time course of cuing effects to evaluate whether inhibition of return can occur in a nonspatial cuing task. Thus, whether inhibition of return effects in spatial cuing tasks and negative validity effects in nonspatial cuing tasks involve the same processes remains an open issue. As an alternative to distinguishing between time course properties of spatial and nonspatial cuing effects, a broad theoretical framework that explains cuing effects in terms not specific to any particular sensory modality or stimulus dimension was offered. Whether or not this particular framework proves useful, the present study suggests that relatively broad processing principles may have an application in even very simple, austere experimental contexts.

\section{REFERENCES}

Bennett, P., \& Pratt, J. (2001). The spatial distribution of inhibition of return. Psychological Science, 12, 76-80.

Bregman, A. S. (1990). Auditory scene analysis. Cambridge, MA: MIT Press.

Collie, A., Maruff, P., Yucel, M., Danckert, J., \& Currie, J. (2000). Spatiotemporal distribution of facilitation and inhibition of return arising from the reflexive orienting of covert attention. Journal of Experimental Psychology: Human Perception \& Performance, 26, 1733-1745.

DANZIGER, S., \& Kingstone, A. (1999). Unmasking the inhibition of return phenomenon. Perception \& Psychophysics, 61, 1024-1037.

Fox, E., \& DeFockert, J. (2001). Inhibitory effects of repeating color and shape: Inhibition of return or repetition blindness? Journal of Experimental Psychology: Human Perception \& Performance, 27, 798812.

JACOBY, L. L. (1978). On interpreting the effects of repetition: Solving a problem versus remembering a solution. Journal of Verbal Learning \& Verbal Behavior, 17, 649-667.

Johnston, W. A., \& HaWley, K. J. (1994). Perceptual inhibition of expected inputs: The key that opens closed minds. Psychonomic Bulletin \& Review, 1, 56-72.

JoNIDES, J. (1981). Voluntary versus reflexive control of the mind's eye's movement. In J. B. Long \& A. D. Baddeley (Eds.), Attention and performance IX (pp. 187-203). Hillsdale, NJ: Erlbaum.

Kahneman, D., Treisman, A., \& GibBs, B. J. (1992). The reviewing of object files: Object-specific integration of information. Cognitive Psychology, 24, 175-219.

KLEIN, R. M. (1988). Inhibitory tagging system facilitates visual search. Nature, 334, 430-431.

KleIn, R. M. (2000). Inhibition of return. Trends in Cognitive Sciences, 4, 138-147.

Klein, R. M., \& Pontefract, A. (1994). Does oculomotor readiness mediate cognitive control of visual attention? Revisited! In C. Umiltà \& M. Moscovitch (Eds.), Attention and performance XV: Conscious and unconscious information processing (pp. 333-350). Cambridge, MA: MIT Press.

Kolers, P. A (1972). Aspects of motion perception. New York: Pergamon.

Kraut, A. G., \& Smothergill, D. W. (1978). A two-factor theory of stimulus-repetition effects. Journal of Experimental Psychology: Human Perception \& Performance, 4, 191-197.

Kwak, H.-W., \& Egeth, H. (1992). Consequences of allocating atten- 
tion to locations and to other attributes. Perception \& Psychophysics, 51, 455-464.

Law, M. B., Pratt, J., \& Abrams, R. A. (1995). Color-based inhibition of return. Perception \& Psychophysics, 57, 402-408.

LogAN, G. D. (1988). Toward an instance theory of automatization. Psychological Review, 95, 492-527.

Lupiáñez, J., Milan, E. G., Tornay, F. J., Madrid, E., \& Tudela, P. (1997). Does IOR occur in discrimination tasks? Yes, it does, but later. Perception \& Psychophysics, 59, 1241-1254.

Lupiáñez, J., Milliken, B., Solano, C., Weaver, B., \& Tipper, S. P. (2001). On the strategic modulation of the time course of facilitation and inhibition of return. Quarterly Journal of Experimental Psychology, 54A, 753-773.

LuPiáÑEZ, J., \& Tudela, P. (1999). Inhibición de retorno al lugar y al color en detección y discriminación/Location- and color-based inhibition of return in a detection task and three different discrimination tasks. Cognitiva, 11, 151-174.

MAYLOR, E. A. (1985). Facilitatory and inhibitory components of orienting in visual space. In M. I. Posner \& O. Marin (Eds.), Attention and performance XI (pp. 189-207). Hillsdale, NJ: Erlbaum.

Milliken,B., Joordens, S., Merikle, P. M., \& Seiffert, A. E. (1998). Selective attention: A reevaluation of the implications of negative priming. Psychological Review, 105, 203-229.

Milliken, B., Lupiáñez, J., Roberts, M., \& Stevanovski,B. (in press). Temporal expectation and spatial orienting: Joint contributions to exogenous spatial cuing effects. Psychonomic Bulletin \& Review.

Mondor, T. A. (1999). Predictability of the cue-target relation and the time-course of auditory inhibition of return. Perception \& Psychophysics, 61, 1501-1509.

Mondor, T. A., Breau, L. M., \& Milliken, B. (1998). Inhibitory processes in auditory selective attention: Evidence of location-based and frequency-based inhibition of return. Perception \& Psychophysics, 60, 296-302.

Mondor, T. A., \& LACY, T. E. (2001). Facilitative and inhibitory effects of cuing sound duration, intensity, and timbre. Perception \& Psychophysics, 63, 726-736.

Mondor, T. A., Zatorre, R. J., \& Terrio, N. A. (1998). Constraints on the selection of auditory information. Journal of Experimental Psychology: Human Perception \& Performance, 24, 66-79.

PALMer, S. E. (1999). Vision science: Photons to phenomenology. London: MIT Press.

PAshler, H., \& BAYlis, G. (1991). Procedural learning: II. Inter-trial repetition effects in speeded-choice tasks. Journal of Experimental Psychology: Learning, Memory, \& Cognition, 17, 33-48.
Posner, M. I. (1980). Orienting of attention. Quarterly Journal of Experimental Psychology, 32, 3-25.

Posner, M. I., \& Cohen, Y. (1984). Components of visual orienting. In H. Bouma \& D. G. Bouwhuis (Eds.), Attention and performance $X$ (pp. 531-556). Hillsdale, NJ: Erlbaum.

Posner, M. I., Rafal, R. D., Choate, L. S., \& Vaughan, J. (1985). Inhibition of return: Neural basis and function. Cognitive Neuropsychology, 2, 211-228.

Rafal, R. D., Calabresi, P. A., Brennan, C. W., \& Sciolto, T. K. (1989). Saccade preparation inhibits reorienting to recently attended locations. Journal of Experimental Psychology: Human Perception \& Performance, 15, 673-685.

SCHNEIDER, W. (1988). Micro Experimental Laboratory: An integrated system for IBM PC compatibles. Behavior Research Methods, Instruments, \& Computers, 20, 206-217.

Tassinari, G., Aglioti, S., Chelazzi, L., Peru, A., \& Berlucchi, G. (1994). Do peripheral non-informative cues induce early facilitation of target detection? Vision Research, 34, 179-189.

TAYLOR, T. L., \& KleIN, R. M. (1998a). Inhibition of return to color: A replication and nonextension of Law, Pratt, and Abrams (1995). Perception \& Psychophysics, 60, 1452-1456.

TAYlor, T. L., \& Klein, R. M. (1998b). On the causes and effects of inhibition of return. Psychonomic Bulletin \& Review, 5, 625-643.

Tipper, S. P., Rafal, R., Reuter-Lorenz, P. A., Starrveldt, Y., Ro, T., Egly, R, Danziger, S., \& Weaver, B. (1997). Object-based facilitation and inhibition from visual orienting in the human splitbrain. Journal of Experimental Psychology: Human Perception \& Performance, 23, 1522-1532.

VAN Selst, M., \& Jolicceur, P. (1994). A solution to the effect of sample size on outlier elimination. Quarterly Journal of Experimental Psychology, 47A, 631-650.

YANTIS, S., \& JonidES, J. (1984). Abrupt visual onsets and selective attention: Evidence from visual search: Journal of Experimental Psychology: Human Perception \& Performance, 10, 601-621.

\section{NOTE}

1. The four groups of participants in Experiment 1 were originally tested in separate experiments. The data are reported here as a single experiment for expository purposes.

(Manuscript received January 29, 2001; revision accepted for publication December 31, 2003.) 